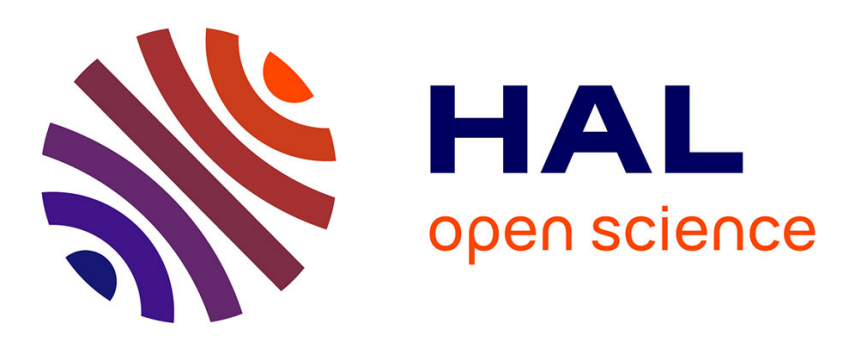

\title{
Evaluation of Biometric Authentication Systems through Visualisation of Partitioned and Bundled Power-graphs
}

Romain Giot

\section{To cite this version:}

Romain Giot. Evaluation of Biometric Authentication Systems through Visualisation of Partitioned and Bundled Power-graphs. 21st International Conference Information Visualisation, Jul 2017, London, France. 10.1109/iV.2017.17 . hal-01575950

\section{HAL Id: hal-01575950 \\ https://hal.science/hal-01575950}

Submitted on 22 Aug 2017

HAL is a multi-disciplinary open access archive for the deposit and dissemination of scientific research documents, whether they are published or not. The documents may come from teaching and research institutions in France or abroad, or from public or private research centers.
L'archive ouverte pluridisciplinaire HAL, est destinée au dépôt et à la diffusion de documents scientifiques de niveau recherche, publiés ou non, émanant des établissements d'enseignement et de recherche français ou étrangers, des laboratoires publics ou privés. 


\title{
Evaluation of Biometric Authentication Systems through Visualisation of Partitioned and Bundled Power-graphs
}

\author{
Romain Giot \\ Labri, Univ. Bordeaux, CNRS, UMR5800, France \\ romain.giot@u-bordeaux.fr
}

\begin{abstract}
Biometric authentication systems verify the identity of individuals based on what they are. As they are error prone, they can reject genuine individuals or accept impostors. Researchers of the field quantify the quality of their algorithm by benchmarking it on several databases. However, although the standard evaluation metrics state the performance of their system, they are unable to explain the reasons of their errors. This paper presents a novel way to visualize the evaluation results of a biometric authentication system which helps to find which individuals or samples are sources of errors. This knowledge could help to fix the algorithms. A biometric database of scores is modeled as a partitioned power-graph with nodes representing biometric samples and power-nodes representing individuals. A novel recursive edge bundling method is also applied to reduce clutter. This proposal has been successfully applied on several biometric databases and has proved its efficiency.
\end{abstract}

\section{INTRODUCTION}

Biometric authentication allows to authenticate individuals based on what they are [1]. Some modalities are common in operational scenarios, as border control the AADHAAR project in India with face [2] or fingerprint recognition [3], while others are more confidential, like keystroke dynamics [4] or veins recognition [5]. A biometric authentication system is composed of two main modules which deeply rely on machine learning and signal processing algorithms (Figure 1): the enrollment module computes the biometric reference of an individual thanks to its enrollment samples in order to model this individual, and the verification module takes the decision to accept the claimant if the matching score computed by comparing its query sample against the claimed biometric reference is higher than the decision threshold (which is a parameter of the biometric authentication system). The machine learning and signal processing methods involved during the different steps executed by the enrollment and verification modules generate errors like false rejection of genuine individuals and false acceptance of impostors. These errors imply the need to evaluate the performance of biometric authentication systems; the standard evaluation consists in selecting a database of biometric samples, specifying an evaluation protocol to produce scores on which various evaluation metrics are applied (see II). Usually, for a database of $I$ individuals with $E+T$ samples per individual, the first $E$ samples of each individual are used to compute its biometric reference, while the $T$ remaining ones are used to compute the verification scores by comparing each sample to each biometric reference.

Although this allows to compare and sort systems to select the best one for an appropriate operational scenario, this does not help to understand which individuals or which samples are at the origin of these errors. The aim of this paper is to present a new interactive visualisation based on partitioned and bundled power-graphs [6] to focus the attention on individuals and samples generating these errors. Thanks to his analysis, the researcher in biometric authentication could better understand the errors reasons and fix the authentication algorithm. The method proposed in this paper shows more information than the Zoograph [7] which has been designed for the same purpose. A power-graph $P G=(V, P V, E V, E P V, E V P V)$ is a graphlike structure containing two kinds of nodes: the nodes $V$ and the power-nodes $P V$ with each power-node representing a strong partition of $V \cup P V . E V$ is the set of edges from one node to another node (the nodes are individually linked), $E P V$ is the set of power-edges from a power-node to another powernode (all the nodes represented by the source power-node are linked to the nodes represented by the target power-node), and $E V P V$ is the set of power-edges from a node to another power-node (the node is linked to all the nodes represented by the power-node). This can be seen as a generalisation of quotient-graphs [8] with the additional ability to link nodes to meta-nodes.

This paper presents two novelties: (i) the method to model and visualize the result of a biometric authentication algorithm, through a partitioned and bundled power-graph, able to highlight the wrong individuals and samples, and (ii) a recursive algorithm to compute edge bundles in a partitioned (powergraph which reduces the edge-node overlap in comparison to standard edge bundling methods by avoiding areas of the screen space reserved to other partitions. Although no contributions are brought to the power-graph [6] representation, this recursive edge bundling method helps to improve their visualisation. The paper is organised as follows. Section II presents common evaluation procedures of biometric authentication systems. Section III and IV respectively explain how to model and visualize a biometric score database with a power-graph to highlight bad behaviors. Section V presents the experimental protocol used to evaluate our contribution, while section VI presents and discusses the results and section VII concludes this paper. 


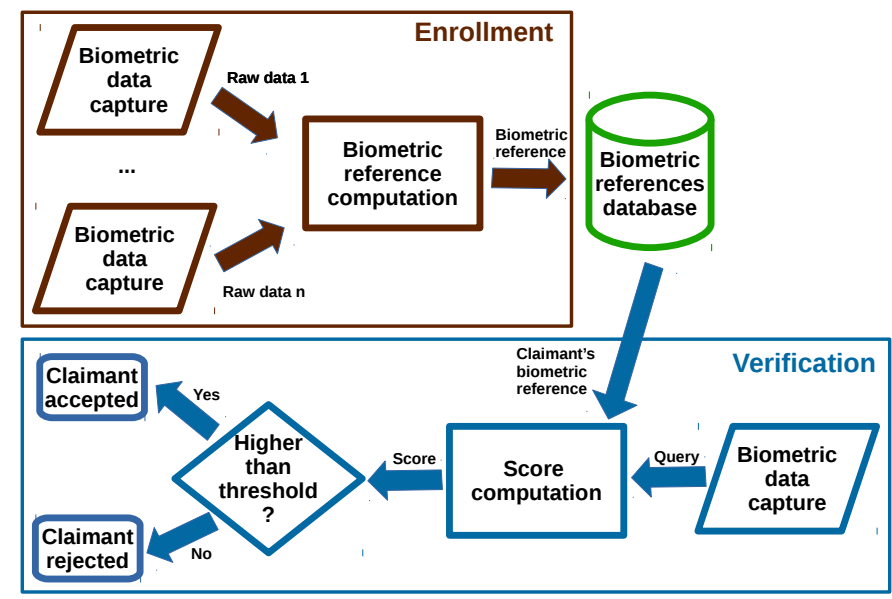

Figure 1. Simplified summary of a biometric authentication system, with its two main modules for enrollment and verification.

\section{Evaluation for Biometric Authentication}

Various evaluation [9] metrics exist to evaluate the authentication performance of a biometric authentication system. The False Match Rate (FMR) is the rate of false acceptance (i.e., samples rejected instead of being accepted) by the matching algorithm for single template comparison attempts (interscores); the False Non Match rate (FNMR) is the rate for false rejection (i.e., samples accepted instead of being rejected) by the matching algorithm for single template comparison attempts (intrascores); finally, the Equal Error Rate (EER) is this error rate of the operational point where FMR and FNMR are equal.

It is also possible to visually evaluate biometric authentication algorithms: the Receiver operating characteristic curve (ROC) [10]: plots the FMR on the $\mathrm{x}$-axis against the corresponding FNMR (more exactly 1-FNMR) on the y-axis depending on the decision threshold; the Zooplot [11] displays all the individuals of the dataset in a scatter plot where their coordinates correspond to their mean genuine and mean impostor scores; the Zoograph [7] improves it by (i) adding an edge between individuals when the source is able, in average, to be recognized as the target and (ii) by using a non linear mapping function on the coordinates ensuring the $25 \%$ best and worst individuals take only $25 \%$ of the screen space each.

Only the Zooplot and the Zoograph provide individual information instead of only giving a global information. The Zoograph goes further by showing the relations between individuals and improving the use of the screen space. However, none of them provide hints on the problematic samples. The power-graph visualisation presented in the remaining sections allows to tackle this issue.

\section{Modeling of Partitioned Power-GRAPHS}

The proposed method (i) models the biometric database of scores with a partitioned power-graph for a specific operational point of threshold $\tau$, and (ii) visualises it through different encoding schemes related to the biometric information.

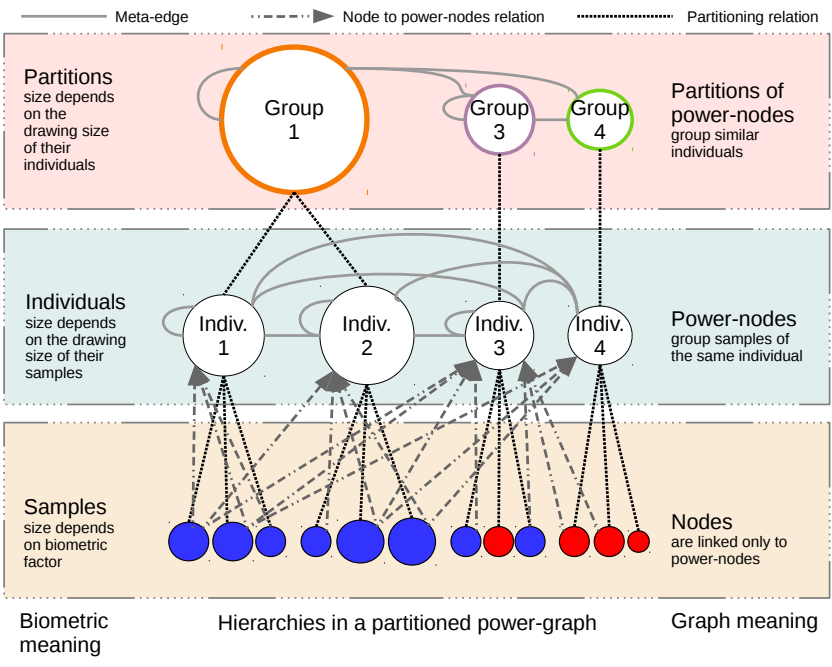

(a) Hierarchies and relations of the simple partitionned power-graph of $2 b$.

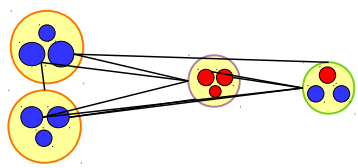

(b) Power-graph before applying edge bundling (powernodes in yellow, nodes in red or blue).

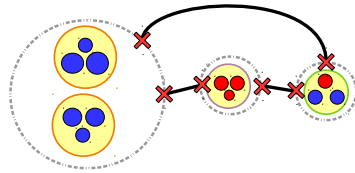

(d) Application of edge bundling at the cluster $\left(Q_{\text {groups }}\right)$ level and addition of artificial intersection nodes (red crosses).

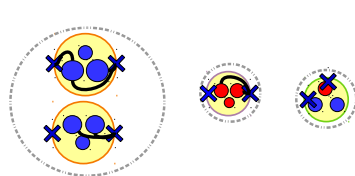

(f) Same process at the sample level.

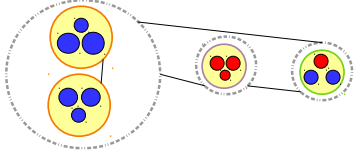

(c) Hierarchical meta-graph representation of the partitionned power-graph (dashed lines represent partitions).

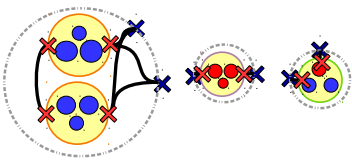

(e) Same process at the individuals ( $\left.Q_{\text {individuals }}\right)$ level; a link between an individual and an artificial node (blue cross) corresponds to an individual linked to another one in another partition.

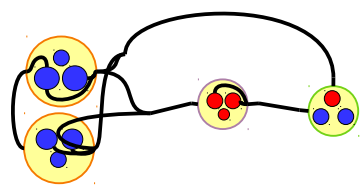

(g) Final result by concatenating the bundles previously computed.
Figure 2. Description of the recursive edge bundling method for power-graphs on a simple partitioned power-graph.

Let's say we have a database of $N$ scores $\mathbb{D}=\cup_{i=1}^{N} t_{i}$, with $t_{i}=\left\{i d_{i}^{\text {probe }}, i d_{i}^{\text {gallery }}\right.$, rank $\left._{i}, s_{i}\right\}$ the information for the $i$ th score; $i d_{i}^{\text {probe }} \in \mathbb{I}$ is the individual who have provided the query, $i d_{i}^{\text {gallery }} \in \mathbb{I}$ is the individual who have provided the biometric reference, $\operatorname{rank}_{i}$ the probe sample number for individual $i d_{i}^{\text {probe }}$ (thus, a sample is identified by the tuple $\left\{i d_{i}^{\text {probe }}, \operatorname{rank}_{i}\right\} \in \mathbb{S}$ ), and $s_{i}$ is the score value of the $i$ th score (i.e., the score obtained by compar- 
ing the sample to the biometric reference). If $i d_{i}^{\text {probe }}=$ $i d_{i}^{\text {gallery }}, s_{i}$ represents an intrascore (otherwise it is an interscore). $\{\mathbb{D}, \tau\}$ can be modeled by a power-graph $P G_{\tau}=$ $\left(\mathbb{S}, \mathbb{I}, \emptyset, \emptyset, \cup_{t_{i} \in \mathbb{D}}\left\{\left\{i d_{i}^{\text {probe }}\right.\right.\right.$, rank $\left.\left.\left._{i}\right\}, i d_{i}^{\text {gallery }}\right\} \mid s_{i} \geq \tau\right)$ where the nodes represent the samples $\mathbb{S}$, the power-nodes represent the individuals $\mathbb{I}$, there is no link between nodes, there is no link between power-nodes, and each node can be linked to power-nodes when the comparison score is higher or equal to $\tau$.

Although $P G_{\tau}$ models relations between samples and individuals, it does not model similarities between individuals. To overcome this issue, its power-nodes are partitioned based on a biometric information computed as follows. Three attributes are computed for each individual $u \in \mathbb{I}$, $F N M R_{\tau}(u)$ its FNMR, $F M R_{\tau}^{i n}(u)$ its FMR when comparing other individuals samples to its own biometric reference, and $F M R_{\tau}^{\text {out }}(u)$ its FMR when comparing all its samples to the biometric reference of all the other individuals. Their average value (among the individuals of the database) are then computed: $F \widehat{N M R_{\tau}}=\sum_{u \in \mathbb{I}} \frac{F N M R_{\tau}(u)}{|\mathbb{I}|}$, $\widehat{F M R_{\tau}^{\text {in }}}=\sum_{u \in \mathbb{I}} \frac{F M R_{\tau}^{\text {in }}(u)}{|\mathbb{I}|}, \widehat{F M R_{\tau}^{\text {out }}}=\sum_{u \in \mathbb{I}} \frac{F M R_{\tau}^{\text {out }}(u)}{|\mathbb{I}|}$. Two thresholds $W$ and $B$ which respectively represent the percentage of errors considered as worst and best performance limits, are used to rank these attributes in 4 categories finally used to compute a partition number for each individual: partition $(u)=\operatorname{score}\left(F \widehat{N M R}_{\tau}, F N M R_{\tau}(u), B, W\right)$ $+\operatorname{score}\left(\widehat{F M R_{\tau}^{\text {out }}}, F N M R_{\tau}^{\text {out }}(u), B, W\right) * 2^{2}$ $+\operatorname{score}\left(\widehat{F M R_{\tau}^{i n}}, F N M R_{\tau}^{i n}(u), B, W\right) * 2^{4}$

where score function result is encoded with 2 bits and defined as score (val,avg, low, high $)=\left\{\begin{array}{l}3, \text { if val }<=\text { low } \\ 2, \text { else if val }<=a v g \\ 1, \text { else if val }<=\text { high } \\ 0, \text { otherwise }\end{array}\right.$

\section{PARTitioned POWER-GRAPH Visualisation}

$P G_{\tau}$ associated to its partitioning information has to be visualised by the biometric authentication researcher to let him understand the behavior of the database amongst his authentication algorithm.

The node-link diagram representation is chosen: its nodes are drawn as discs and its power-nodes as rounded squares; by definition, each node is drawn on top of a power-node. Several information need to be shown in evidence in order to ease the comprehension of the displayed structure.

Individuals proximity: individuals of a same partition have to be drawn close together. We first build the quotient-graph [8] $Q_{\text {individuals }}$ of the graph of samples which represents the individuals of the database: one meta-node per individual, one meta-edge between two individuals if one of them have samples impersonating the other one. We then build the quotient graph $Q_{\text {groups }}$ based on the individuals partitions: one meta-node per partition of individuals, one meta-edge between two metanodes if one individual of the source partition is linked to one individual of the target partition. Thanks to this 3 levels hierarchy, a bottom-up multilevel drawing method is used to compute the embedding of the nodes and power-nodes of $P G_{\tau}$. (1) The samples subgraph of each individual is drawn with a packing algorithm and its size is reported to the corresponding node in $Q_{\text {individuals. }}$ (2) The individuals subgraph of each partition of individual is drawn by using an algorithm which ensures no node-node overlap and its size is reported to the corresponding node in $Q_{\text {partition }}$. (3) $Q_{\text {partition }}$ is drawn by using an algorithm ensuring no nodenode overlap. (4) The absolute position of each node of $P G_{\tau}$ is trivially computed by recursively centering partitions to the coordinate of is meta-node representative from the top of the hierarchy to the bottom. Figure 2 a summarizes the structures involved in the drawing with a simple partitioned power-graph (Figure2b). Individual node color corresponds to $H S V\left(F N M R \_s c o r e, F M R \_s c o r e^{i n}, F M R \_s c o r e^{o u t}\right)$ at a scale factor precision.

Intraclass performance: if a sample is detected to be genuine, its is drawn in blue, otherwise in red. It allows to quickly identify samples rejected as well as individuals having more rejected samples than the others. The border color of an individual corresponds to $R G B\left(F N M R_{\tau}(u), 0,1-F N M R_{\tau}(u)\right)$.

Interclass performance: the size of each sample depends on its ability to impersonate other individuals (bigger means more errors). By construction it impacts the size of the drawing of power-nodes.

Edge-bundling algorithms can reduce the clutter due to edges drawn over the nodes and power nodes. However, standard edge bundling methods allow edges to route between nodes belonging to partitions different than the source and destination ones. To overcome this issue, we have thus designed a new recursive edge-bundling method able to avoid partitions. The first step computes the edge bundling of $Q_{\text {groups }}$ while ensuring the first (respectively last) bend of each meta-edge is on the perimeter of the source (respectively target) meta-node and adds an artificial node in the subgraph represented by each meta-node at the intersection position (Figure 2d). An artificial edge is added between the source (respectively artificial) node and the artificial (respectively target) node in the subgraph represented by the source (respectively target) meta-node for each edge represented by a meta-edge. The next step applies the same procedure to the subgraph represented by each meta-node of $Q_{\text {groups }}$ (Figure 2e). In the next step, edge bundling is then computed on the induced subgraph represented by each each meta-node of $Q_{\text {individuals }}$ (Figure 2f). Finally, the bends of each edge of the power-graph correspond to the concatenation of the bends computed in the previous steps (Figure $2 \mathrm{~g}$ ).

As the embedding of $P G_{\tau}$ can be huge, it has to be viewed in an interactive (mainly zooming and panning) application able to highlight interactions between samples and individuals. This is achieved thanks to a neighbourhood interactor [17]: to select an individual highlights the samples able to impersonate him while to select a sample highlights individuals it is able to impersonate. To reduce the visual noise, the part of an edge inside a power-node is only drawn when this edge is highlighted by the neighbourhood interactor. 
Table I

DESCRIPTION OF THE DATASETS USED TO EVALUATE THE PROPOSAL.

\begin{tabular}{|c|c|c|c|c|c|c|c|}
\hline Dataset & Type & Modality & Methodology & \# individuals & $\begin{array}{c}\text { \# sample } \\
\text { /individuals }\end{array}$ & \# scores & EER \\
\hline AR [12] & Score & Face & SIFT based matching & 120 & 26 & 360000 & $10.19 \%$ \\
\hline ENSIB [13] & Score & Face & SIFT based matching & 100 & 40 & 390000 & $10.88 \%$ \\
\hline FC94 [14] & Score & Face & SIFT based matching & 152 & 20 & 438976 & $0.29 \%$ \\
\hline FVC [15] & Score & Fingerprint & SIFT based matching & 100 & 8 & 70000 & $10.27 \%$ \\
\hline veins [5] & Score & Vein & SIFT based matching & 24 & 30 & 16704 & $0.0 \%$ \\
\hline OU-ISIR BSS3 [16] & Distance & $\begin{array}{l}\text { Gait } \\
\text { (accelerometer) }\end{array}$ & $\begin{array}{l}\text { Distance between } 2 \text { sig- } \\
\text { nals }\end{array}$ & 736 & variable & 10175181 & $14.88 \%$ \\
\hline
\end{tabular}

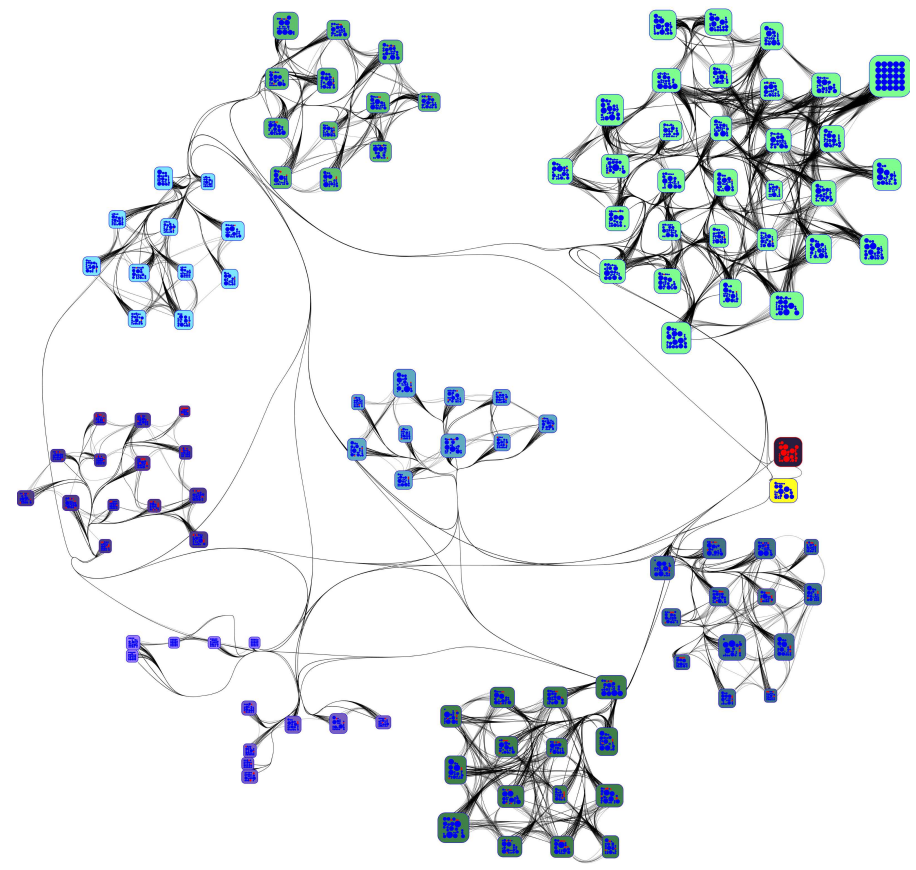

(a) Result on AR.

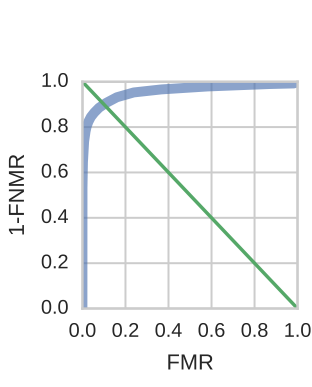

(c) ROC curve on AR.

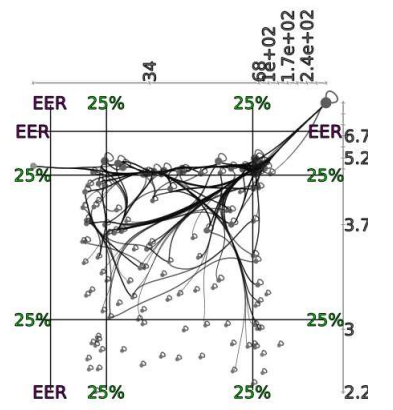

(d) Zoograph on AR.

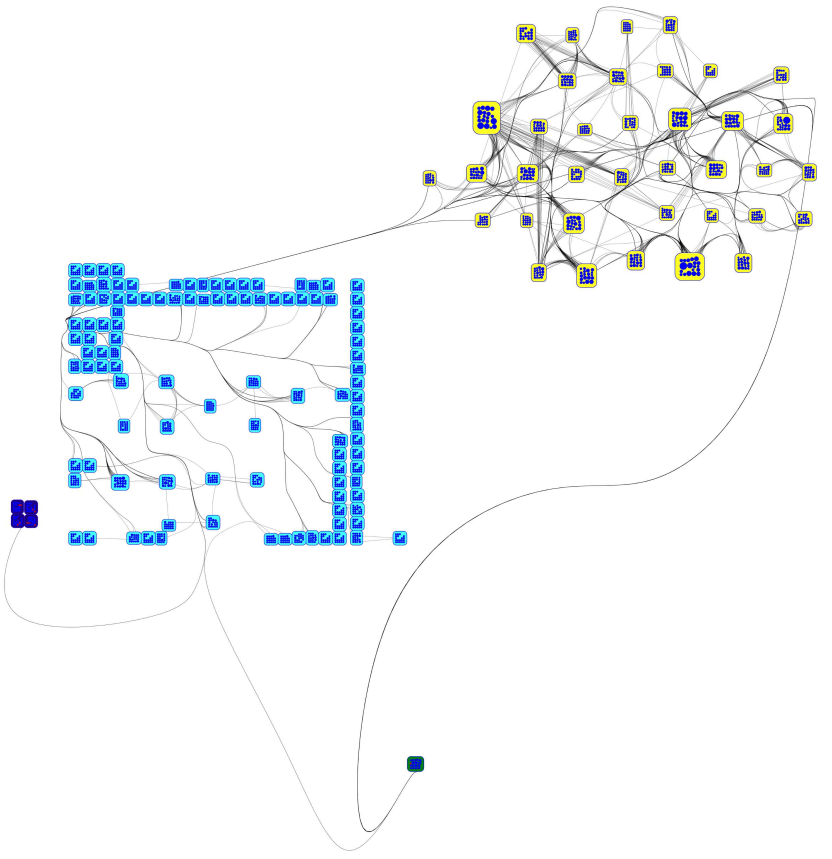

(b) Result on FC94.

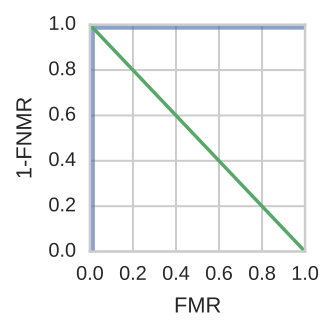

(e) ROC curve on FC94.

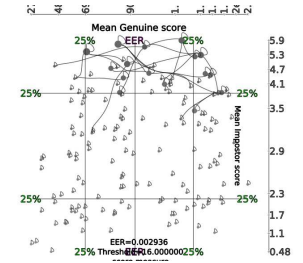

(f) Zoograph on FC94.

Figure 3. Comparison of the visualisation produced by the proposed method against the baselines. (continues)

\section{EXPERIMENTAL PROTOCOL}

Table I presents the datasets of scores used to evaluate the proposal; the number of individuals (it also corresponds to the number of power-nodes) is in the range [24;736], the number of scores (it also corresponds to the maximum number of edges) is in the range $[16704 ; 10175181]$, while the EER is in the range $[0 ; 0,1488]$. The threshold $\tau$ is configured to correspond to the EER operational point. $W$ and $B$ are respectively set to $80 \%$ and $5 \%$. Among the different evaluation methodologies of the literature, we want to compete with the Zoograph [7] which is used as the baseline for local evaluation method, while the ROC curve is used as the baseline for global evaluation method. The proof of concept is written with around 4000 lines of 


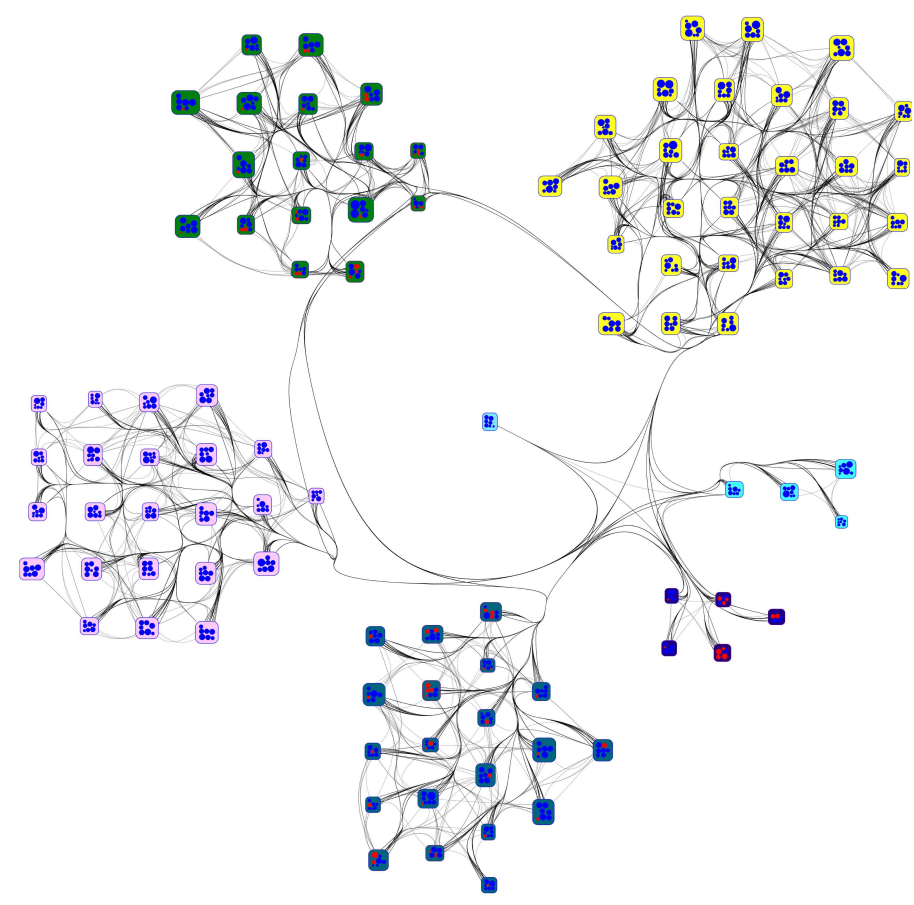

(g) Result on FVC.

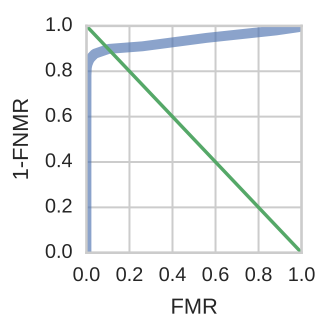

(i) ROC curve on FVC.

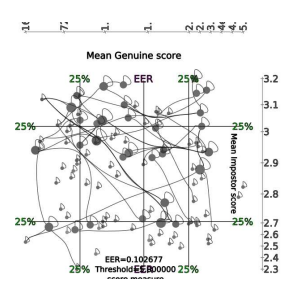

(j) Zoograph on FVC.

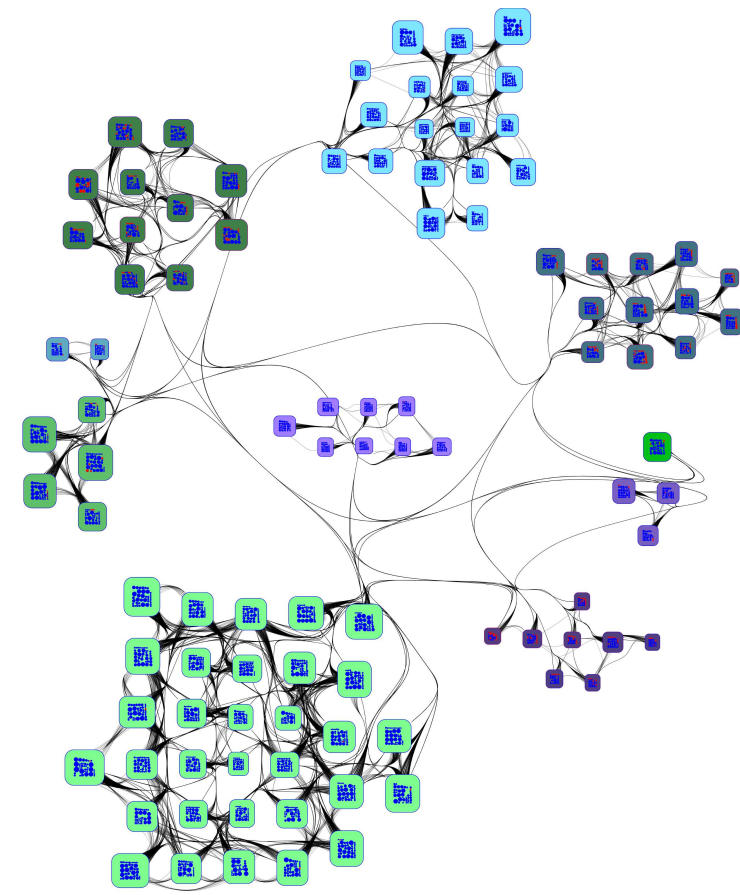

(h) Result on ENSIB.

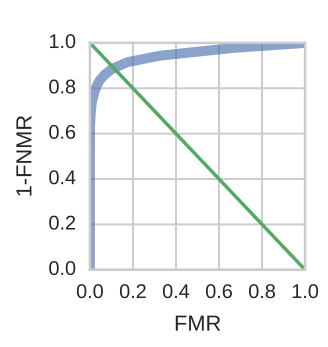

(k) ROC curve on ENSIB.

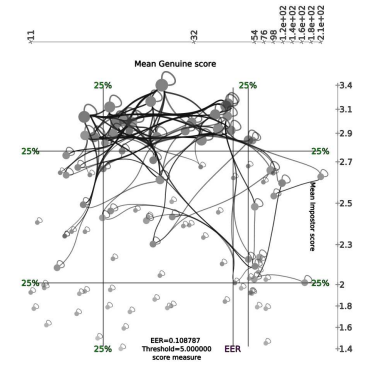

(1) Zoograph on ENSIB .

Figure 3. continued

$\mathrm{C}++14$ and intensively uses the graph display library Tulip 4.11 [18] and its associated plugins; each partition of samples is drawn with GRIP [19], each partition of individual is drawn with Fast Multipole Embedder [20] followed by Fast Node Overlap Removal [21] then followed by a connected component packing method to drastically reduce the drawing size of good partitions; $Q_{\text {groups }}$ is drawn with Bubble Tree [22]; finally, the bundling algorithm used by the recursive bundling algorithm is Winding Roads [23]. The edges are drawn with cubic B-splines in black color with an alpha value of $100 / 255$ in order to emphasize the positions with a high edge density (which is frequent due to the edge bundling).

\section{RESULTS}

Figure 3 gives a still representation of the power-graph drawn for each evaluated dataset and compares them to the ROC curve and the Zoograph. It clearly provides more information than the ROC curves; for example, on the system FC94 which is almost perfect ( $0.29 \%$ of errors), we easily detect the few individuals at the origin of these false acceptances (upperright group) as well as the individuals having false rejections (left group). This representation lies less than the Zoograph as we see more individuals linked together (ENSIB, FC94) because a sample is linked to an individual if there is a match, whereas for the Zoograph two individuals are linked if there is a match in average. In the Zoograph, individuals have links only if they do errors in average which hides more diffuse errors. The partitioning of individuals thanks to a biometric information helps to see if there are different categories of individuals. Although the number of categories varies among the datasets, it is always lower than the theoretical maximal number of partitions $\left(4^{3}=64\right)$. Figure 4 presents the drawing result of the AR database without bundling and with standard edge bundling. It shows that: (i) using a bundling method is mandatory as the number of edges is too much important even for systems with average error rates (10\%), and (ii) the non-recursive edge bundling allows edges to cross partitions which complicates the understanding of the 


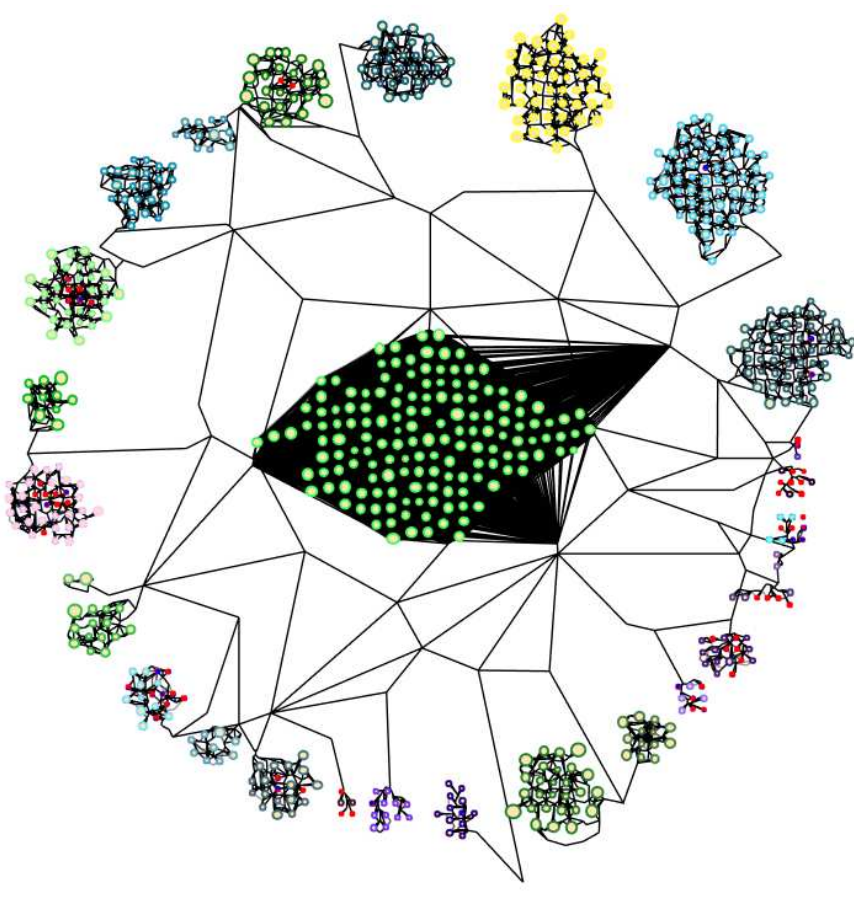

(m) Result on BSS3 (edges represented with polylines for memory reasons).

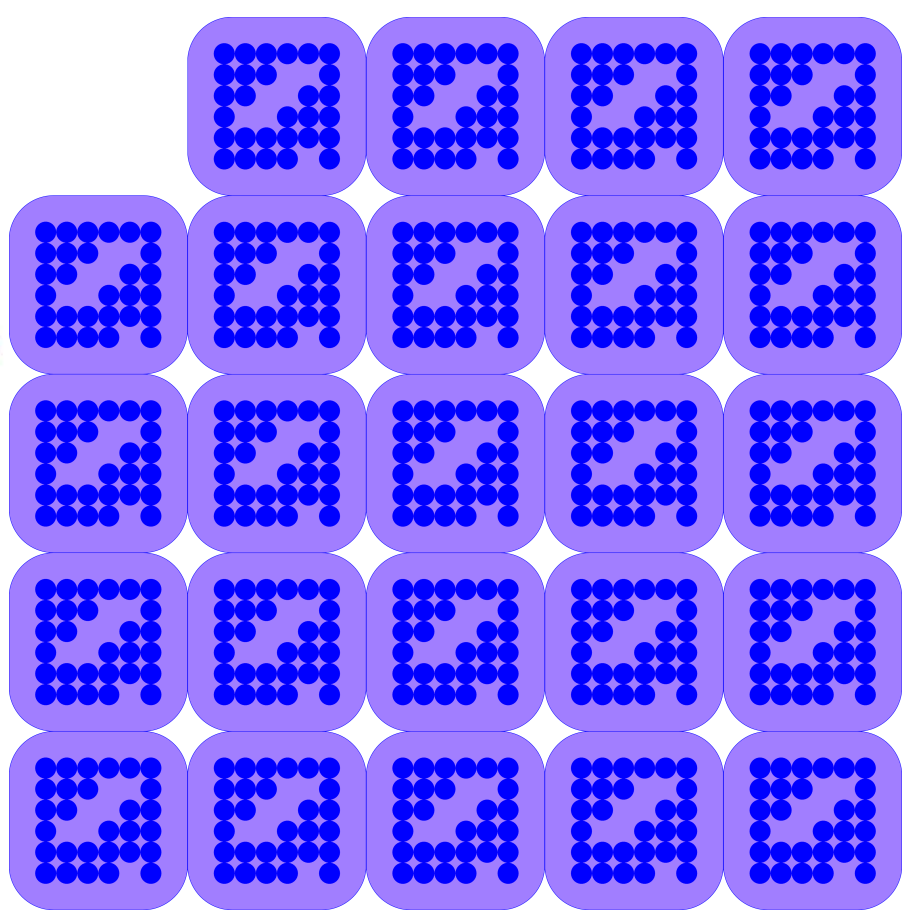

(n) Result on veins.

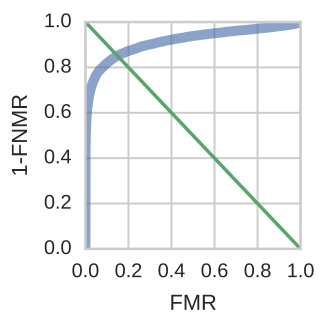

(o) ROC curve on BSS3.

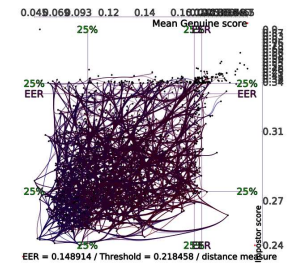

(p) Zoograph on BSS3.

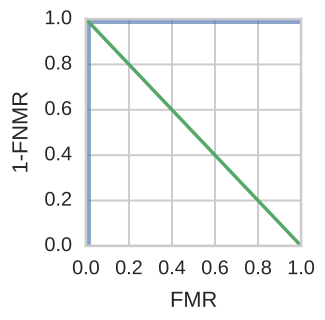

(q) ROC curve on veins.

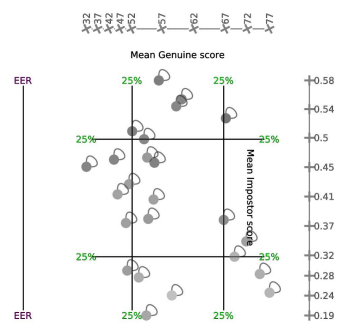

(r) Zoograph on veins.

Figure 3. continued

drawing. The novel recursive edge-bundling is indisputably an interesting contribution for such drawing. Figure 5 presents two interactions implemented: (i) the highlight of the samples able to impersonate the selected individual, and (ii) the highlights of the individuals impersonated by the selected sample. These two interactions are mandatory to explore the result graph.

\section{CONCLUSION}

As biometric authentication systems are error prone, researchers have to evaluate them with benchmark datasets. The main evaluation methods of the literature provide global evaluation metrics and visualisation without emphasising on the samples or individuals sources of errors. This paper presented a novel visualisation method which encodes the result of the evaluation of a biometric authentication system as a powergraph, computes its embedding after partitioning its powernodes and uses a novel recursive edge-bundling method to reduce noise. Results are promising as this new visualisation displays something novel: the samples sources of errors. The recursive edge-bundling method developed for this purpose is also a great addition to reduce the noise and can be easily adapted to work on any hierarchically partitioned graph. We have identified several improvements which are left for future work. To display the biometric images of morphological modalities instead of shapes would allow to better understand the reasons of mistakes (for examples if the rejected samples all corresponds to blurry images). Confluent drawings [24], which does bundling based on a power-graph representation, could also be an alternative to the recursive edge bundling after adding constraints ensuring no partition traversal.

\section{ACKNOWLEDGMENTS}

Thanks to Dr. Mohamad El-Abed for having computed and provided several databases of scores.

\section{REFERENCES}

[1] A. Jain, P. Flynn, and A. A. Ross, Handbook of biometrics. Springer Science \& Business Media, 2007.

[2] A. K. Jain and S. Z. Li, Handbook of face recognition. Springer, 2011. 


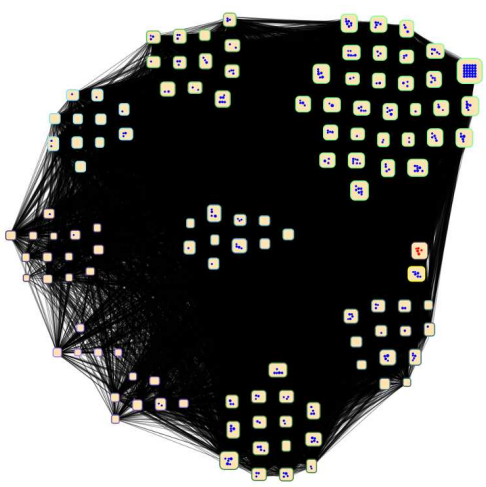

(a) AR dataset with no edge bundling.

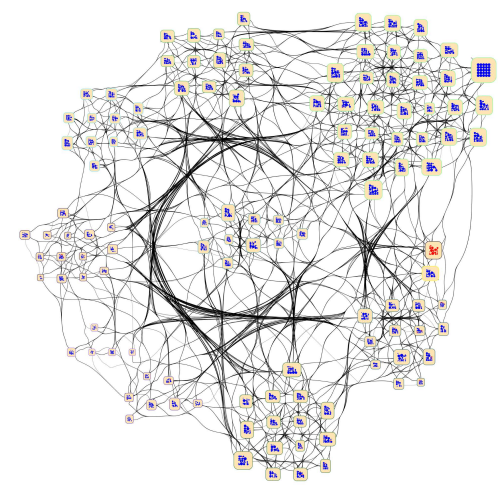

(b) AR dataset with standard edge bundling.

Figure 4. Visualisation without the proposed recursive edge bundling method.

[3] D. Maltoni, D. Maio, A. Jain, and S. Prabhakar, Handbook of fingerprint recognition. Springer Science \& Business Media, 2009.

[4] R. Giot, M. El-Abed, and C. Rosenberger, "Keystroke dynamics overview," in Biometrics. InTech, 2011.

[5] P.-O. Ladoux, C. Rosenberger, and B. Dorizzi, "Palm vein verification system based on sift matching," in the 3rd IAPR/IEEE International Conference on Biometrics (ICB'09), 2009, pp. 1290-1298.

[6] L. Royer, M. Reimann, B. Andreopoulos, and M. Schroeder, "Unraveling protein networks with power graph analysis," PLoS Comput Biol, vol. 4, no. 7, p. e1000108, 2008.

[7] R. Giot, R. Bourqui, and M. El-Abed, "Zoo graph: a new visualisation for biometric system evaluation," in Information Visualisation 2016, 2016, pp. 190-195.

[8] R. Brockenauer and S. Cornelsen, "Drawing clusters and hierarchies," in Drawing graphs. Springer, 2001, pp. 193-227.

[9] ISO/IEC 19795-1, Information technology - biometric performance testing and reporting - Part 1: Principles and framework, International Organization for Standardization tt Std., 2006.

[10] J. R. Matey, G. W. Quinn, P. Grother, E. Tabassi, C. Watson, and J. L. Wayman, "Modest proposals for improving biometric recognition papers," in Biometrics Theory, Applications and Systems, 2015 IEEE 7th International Conference on, 2015, pp. 1-7.

[11] N. Yager and T. Dunstone, "The biometric menagerie," Pattern Analysis and Machine Intelligence, IEEE Transactions on, vol. 32, no. 2, pp. 220-230, 2010.

[12] A. Martinez and R. Benavente, "The AR face database," CVC Tech. Report, 1998. [Online]. Available: http://www2.ece.ohiostate.edu/ aleix/ARdatabase.html

[13] B. Hemery, C. Rosenberger, and H. Laurent, "The ENSIB database : a benchmark for face recognition," in International Symposium on Signal Processing and its Applications, special session "Performance Evaluation and Benchmarking of Image and Video Processing”, 2007.

[14] U. of Essex, "Faces94 database, face recognition data," 1994.

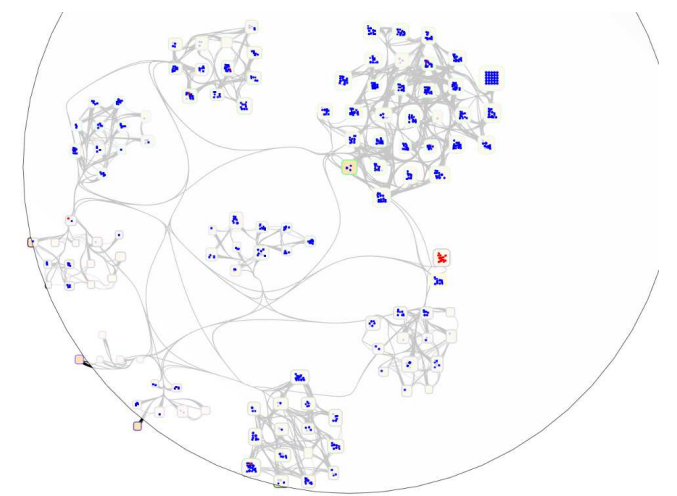

(a) Interaction with an individual (top right partition).

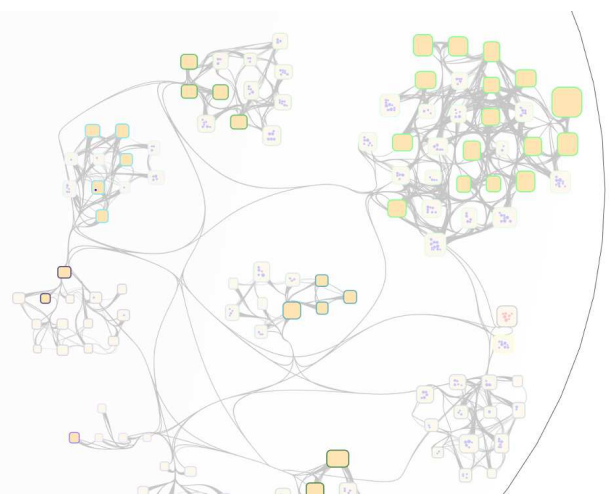

(b) Interaction with a sample (middle left cluster)

Figure 5. Highlights of the interactions.

[15] D. Maio, D. Maltoni, R. Cappelli, J. L. Wayman, and A. K. Jain, "Fvc2002: Second fingerprint verification competition," in International Conference on Pattern Recognition, vol. 3, 2002, pp. 811 - 814. [Online]. Available: http://bias.csr.unibo.it/fvc2002/

[16] N. T. Trung, Y. Makihara, H. Nagahara, Y. Mukaigawa, and Y. Yagi, "Performance evaluation of gait recognition using the largest inertial sensor-based gait database," in Biometrics, 2012 5th IAPR International Conference on, 2012, pp. 360-366.

[17] T. Moscovich, F. Chevalier, N. Henry, E. Pietriga, and J.-D. Fekete, "Topology-Aware Navigation in Large Networks," in SIGCHI conference on Human Factors in computing systems, A. Press, Ed., Boston, ÉtatsUnis, 2009, pp. 2319-2328.

[18] D. Auber, D. Archambault, R. Bourqui, M. Delest, J. Dubois, B. Pinaud, A. Lambert, P. Mary, M. Mathiaut, and G. Melancon, "Tulip III," in Encyclopedia of Social Network Analysis and Mining. Springer, 2014, pp. 2216-2240.

[19] P. Gajer and S. Kobourov, "Grip: Graph drawing with intelligent placement," in Graph Drawing. Springer, 2001, pp. 104-109.

[20] M. Gronemann, "Engineering the fast-multipole-multilevel method for multicore and simd architectures," Master's thesis, Technische Universität Dortmund, 2009.

[21] T. Dwyer, K. Marriott, and P. J. Stuckey, "Fast node overlap removal," in International Symposium on Graph Drawing, 2005, pp. 153-164.

[22] S. Grivet, D. Auber, J.-P. Domenger, and G. Melancon, "Bubble tree drawing algorithm," in Computer Vision and Graphics. Springer, 2006, pp. 633-641.

[23] A. Lambert, R. Bourqui, and D. Auber, "Winding roads: Routing edges into bundles," in Computer Graphics Forum, vol. 29, no. 3, 2010, pp. 853-862.

[24] B. Bach, N. H. Riche, C. Hurter, K. Marriott, and T. Dwyer, "Towards unambiguous edge bundling: Investigating confluent drawings for network visualization," in IEEE Transactions on Visualization and Computer Graphics, 2016, pp. 541-550. 